\title{
Vertical modeling of a quadcopter for mass estimation and diagnosis purposes
}

Du Ho, J onas Linder, Gustaf Hendeby and Martin Enqvist

\section{Conference article}

N.B.: When citing this work, cite the original article.

Part of: Proceedings of the Workshop on Research, Education and Development on Unmanned Aerial Systems, RED-UAS, Linköping, Sweden, 3-5 October, 2017, ISBN: 978-1-5386-0939-2 (electronic), 978-1-5386-0940-8 (print) doi.org/ 10.1109/RED-UAS.2017.8101665

Copyright: www.ieee.org

Available at: Linköping University Institutional Repository (DiVA)

http:/ / urn.kb.se/ resolve?urn=urn:nbn:se:liu:diva-141883

\section{$\Rightarrow$ Tweet}




\title{
Vertical modeling of a quadcopter for mass estimation and diagnosis purposes
}

\author{
Du Ho ${ }^{1}$, Jonas Linder ${ }^{1}$, Gustaf Hendeby ${ }^{1}$ and Martin Enqvist ${ }^{1}$
}

\begin{abstract}
In this work, we estimate a model of the vertical dynamics of a quadcopter and explain how this model can be used for mass estimation and diagnosis of system changes. First, a standard thrust model describing the relation between the calculated control signals of the rotors and the thrust that is commonly used in literature is estimated. The estimation results are compared to those using a refined thrust model and it turns out that the refined model gives a significant improvement. The combination of a nonlinear model and closed-loop data poses some challenges and it is shown that an instrumental variables approach can be used to obtain accurate estimates. Furthermore, we show that the refined model opens up for fault detection of the quadcopter. More specifically, this model can be used for mass estimation and also for diagnosis of other parameters that might vary between and during missions.
\end{abstract}

\section{INTRODUCTION}

The use of small aerial vehicles and especially quadcopters has been growing rapidly in the past few years. Reasons for this include the quadcopter's relatively simple mechanical structure and its high maneuverability. A standard quadcopter has four symmetrically placed propellers with fixed pitch that are arranged in counter-rotating pairs. The rotors generate a vertical thrust and three torques corresponding to the three rotation axes, which allows the quadcopter to perform quick and complex maneuvers. Another reason for the popularity of the quadcopter is its ability to carry a variety of payloads, which allows it to perform different tasks.

Recent technological developments in sensors, batteries and microcomputers/microcontrollers allow for autonomous flight using quadcopters at a reasonable cost. Therefore, single and cooperating quadcopters have been used in various applications, such as surveillance, search and rescue missions [1] and exploration and mapping of 3D environments [2], [3].

A quadcopter is a inherently unstable and underactuated system [4], which makes manual control very difficult. This is handled using an on-board controller that stabilizes the quadcopter. The performance of the controller depends heavily on the quadcopter's state and its surrounding environments, such as the attitude, altitude and so on; therefore, the sensing system is an important research topic [5]. However, adding more on-board sensors, such as a global positioning system (GPS) device or camera, increases the weight of the payload and reduces the maneuverability, and may even cause the quadcopter to crash. It can therefore be interesting

\footnotetext{
${ }^{1}$ Division of Automatic Control, Department of Electrical Engineering, Linköping University, SE-58183 Linköping, Sweden. Email: \{du.ho.duc, jonas.linder, gustaf.hendeby, martin.enqvist\}@liu.se
}

to monitor the payload, to allow the quadcopter to land safely if needed.

Moreover, various types of more challenging flight scenarios have been reported in recent years, e.g., aggressive flight maneuvers, such as spins and flips [6] and dancing in the air [7]. During these aggressive flights, the quadcopters are affected by various aerodynamic effects, such as blade flapping [8] and thrust variation [9] due to changes in the induced velocities. Hence, performing extreme maneuvers result in high power consumption peaks and overall high power consumption, which drains the battery capacity rapidly. The impacts of aerodynamics and battery lifetime need to be taken into consideration in the design of the electronic control unit (ECU). In particular, any change in the thrust generation has to be detected and compensated for. More precisely, to allow autonomous aerial vehicles to deal with unexpected incidents, it is necessary to detect changes/faults in the system before they lead to a complete system breakdown [10]. Therefore, it is for example necessary to track the parameter changes in the thrust model, which reflect actuator lock and other potential actuator problems [11].

In [12], a drag-force enhanced dynamic model is used to detect changes in the mass of the quadcopter. This model includes the flapping effect, which describes how the propeller interacts with the air. The approach only focuses on horizontal flight, which can occur during particular flight segments of a mission. However, a mission usually does not only contain horizontal flight between waypoints with constant altitude, but also segments of vertical flight with stationary attitude. During these segments of maneuver, system identification algorithms could be applied to estimate approximately the physical parameters of the quadcopter [13], [14]. Therefore, this work extends the previous work in [12] for the latter case. Firstly, a refined version of the standard lumped parameters model for the propeller aerodynamic of the quadcopter is derived. Furthermore, the standard model and a refined model of the thrust are compared. The parameters of the two models are estimated based on the collected data using low-cost on-board sensors. This limited sensor setup complicates the estimation problem compared to the work of [15] where several additional sensors such as motor angular speed sensors are available. Finally, the possibilities to perform battery and weight diagnosis by detecting changes in the parameters of the model are investigated.

The paper outline is as follows. In Sec. II, the refined aerodynamic model describing the relation between the calculated control signal and the thrust of the quadcopter is presented, and one standard and one refined vertical model 


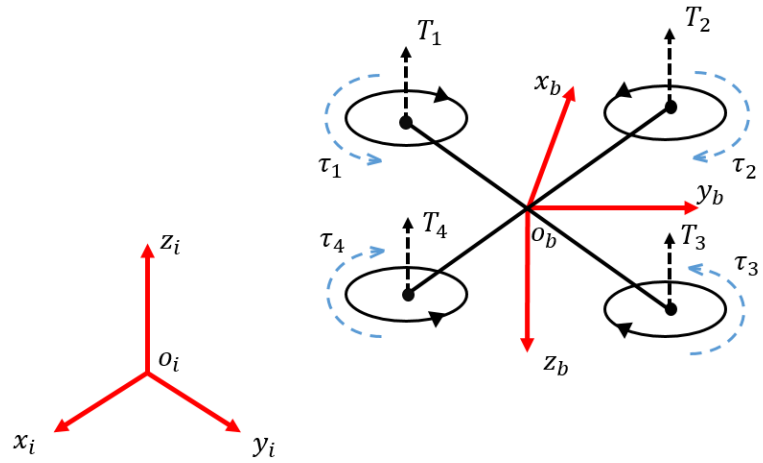

Fig. 1. The inertial and the body coordinate frames of the quadcopter.

are formulated. The experimental parameter estimation results of the standard and refined models are presented in Sec. III. Sec. IV describes the mass change detection using both models and a comparison with previous work. The discussion about diagnosis of the quadcopter is given in Sec. V, and Sec. VI concludes the paper.

\section{Modeling}

In this section, the mathematical equation of the translational model of a quadcopter is presented and a subsystem is considered for estimation purposes.

\section{A. Quadcopter dynamics}

Consider a quadcopter as in Fig. 1. The position of the quadcopter in the inertial frame is defined as $\boldsymbol{\xi}_{i}=$ $\left[\begin{array}{lll}x_{i} & y_{i} & z_{i}\end{array}\right]^{T}$. The orientation of the quadcopter is described by three attitude Euler angles which are denoted by $\boldsymbol{\eta}=$ $\left[\begin{array}{lll}\phi & \theta & \psi\end{array}\right]^{T}$.

The origin of the body frame is chosen to coincide with the quadcopter's center of mass. In the body frame, $\boldsymbol{V}_{b}=$ $\left[\begin{array}{lll}u & v & w\end{array}\right]^{T}$ and $\boldsymbol{v}=\left[\begin{array}{lll}p & q & r\end{array}\right]^{T}$ define the translational and the angular velocities, respectively.

The relation between the translational velocities in the body-fixed frame and those in the inertial frame is described by the rotation matrix

$$
\boldsymbol{R}=\left[\begin{array}{ccc}
C_{\theta} C_{\psi} & S_{\phi} S_{\theta} C_{\psi}-C_{\phi} S_{\psi} & C_{\phi} S_{\theta} C_{\psi}+S_{\phi} S_{\psi} \\
C_{\theta} S_{\psi} & S_{\phi} S_{\theta} S_{\psi}+C_{\phi} C_{\psi} & C_{\phi} S_{\theta} S_{\psi}-S_{\phi} C_{\psi} \\
-S_{\theta} & S_{\phi} C_{\theta} & C_{\phi} C_{\theta}
\end{array}\right]
$$

where $S_{x}=\sin x$ and $C_{x}=\cos x$.

The quadcopter is assumed to be a rigid body and thus the Newton-Euler equations can be used to describe its dynamics. In the body-fixed frame, the translational motion equation of the quadcopter is given by

$$
m \dot{\boldsymbol{V}}_{b}+\boldsymbol{v} \times\left(m \boldsymbol{V}_{b}\right)=m \boldsymbol{R}^{T} \boldsymbol{g}+\boldsymbol{T}_{b}-\boldsymbol{\lambda} \boldsymbol{V}_{b},
$$

where $m$ is the mass of the quadcopter and the other variables and constants are explained in Table I.

Projecting (2) onto the $z_{b}$ axis yields

$$
\dot{w}=-\frac{T_{z}}{m}-\frac{k_{w}}{m} w+g \cos \theta \cos \phi .
$$

TABLE I

THE NOTATION OF THE TRANSLATIONAL DYNAMIC EQUATION.

\begin{tabular}{|r|l|}
\hline Symbol & Quantity \\
\hline$m$ & Mass of the quadcopter $[\mathrm{kg}]$ \\
\hline$\dot{\boldsymbol{V}}_{b}$ & Accelerations of the quadcopter $\left[\mathrm{m} / \mathrm{s}^{2}\right]$ \\
\hline $\boldsymbol{V} \times\left(m \boldsymbol{V}_{b}\right)$ & The centrifugal force $[\mathrm{N}]$ \\
\hline $\boldsymbol{g}$ & The gravity vector $\left[\mathrm{m} / \mathrm{s}^{2}\right]$ \\
\hline $\boldsymbol{R}$ & The rotation matrix \\
\hline $\boldsymbol{T}_{b}$ & The total thrust $[\mathrm{N}]$ \\
\hline $\boldsymbol{\lambda}$ & The drag coefficient matrix $[\mathrm{Ns} / \mathrm{m}]$ \\
\hline
\end{tabular}

The inertial measurement unit (IMU) consists of an accelerometer and a gyroscope which measure the linear accelerations and the angular velocities in three dimensions in a sensor-fixed frame. The sensor-fixed coordinate frame is assumed to coincide with the body-fixed frame except for a $180^{\circ}$ rotation around the $x_{b}$ axis and the vertical acceleration measurement is

$$
a_{z}=\frac{T_{z}}{m}+\frac{k_{w}}{m} w+e_{a_{z}} .
$$

The quadcopter has fixed-pitch propellers and the motors are assumed to produce the force

$$
\boldsymbol{T}_{b}=\left[\begin{array}{lll}
0 & 0 & T_{z}
\end{array}\right]^{T},
$$

where $T_{z}$ is computed as $T_{z}=\sum_{i=1}^{4} T_{i}$. The standard model of the thrust produced by the $i^{\text {th }}$ propeller is given by $T_{i}=k_{\omega} \omega_{i}^{2}$, where $\omega_{i}$ is the angular speed of the $i^{\text {th }}$ propeller and $k_{\omega}$ is a positive constant. A better dynamic model that more accurately explains the underlying physics using bladeelement momentum theory is given in [15], [16] and is described by

$$
T_{i}=c_{1} \omega_{i}^{2}\left(c_{2}\left(1+\frac{3}{2} \mu_{i}^{2}\right)-\lambda_{i}\right)
$$

where $c_{1}$ and $c_{2}$ are positive constants which depend on the propeller's structure. The advance ratio and inflow ratio are

$$
\mu_{i}=\frac{V_{h i}+v_{h i}}{R \omega_{i}}
$$

and

$$
\lambda_{i}=\frac{V_{z i}+v_{z i}}{R \omega_{i}}
$$

respectively, where $R$ is the radius of the propeller, $V_{h i}$ and $V_{z i}$ are the horizontal and vertical velocities of the $i^{\text {th }}$ rotor in the body-fixed frame, and $v_{h i}$ and $v_{z i}$ are the horizontal and vertical induced velocities of the air stream through the $i^{\text {th }}$ rotor, respectively.

During vertical flight maneuvers, the quadcopter is controlled to fly vertically with zero roll and pitch angles. It is assumed that the horizontal speed $V_{h i} \approx 0$ and that the horizontal induced velocity $v_{h i} \approx 0$ while the vertical speeds are assumed to be constant. This implies that the force from the $i^{\text {th }}$ motor can be approximated by

$$
T_{i}=\bar{c}_{1} \omega_{i}^{2}+\bar{c}_{2} \omega_{i}
$$

Assuming that the dynamics of the rotors are fast enough to be neglected, the relation between the calculated control 
signal and the angular speed, that is associated with the ECU, is given by $\omega_{i}=k_{\mathrm{c}} u_{\mathrm{ci}}$. Moreover, all rotors are assumed to be identical, which implies that the vertical force is given by

$$
T_{z}=\sum_{i=1}^{4} k_{1} u_{\mathrm{ci}}^{2}+k_{2} u_{\mathrm{ci}}=k_{1} u_{i n 1}+k_{2} u_{i n 2}
$$

where $k_{1}$ and $k_{2}$ are positive and unknown constants. In this work, we will also consider the standard thrust model given by $T_{z}=\sum_{i=1}^{4} k_{1} u_{\mathrm{ci}}^{2}=k_{1} u_{i n 1}$.

\section{B. Model approximation}

Combining (3), (4) and (9), and assuming small angles $(\cos (\phi) \approx \cos (\theta) \approx 1)$, the transfer function from $u_{\text {in } 1}$ and $u_{\text {in } 2}$ to $a_{z}$ is given by

$$
a_{z}=\frac{\mathrm{p}}{\mathrm{p}+\frac{k_{w}}{m}}\left(\frac{k_{1}}{m} u_{i n 1}+\frac{k_{2}}{m} u_{i n 2}\right)+\frac{\frac{k_{w} g}{m}}{\mathrm{p}+\frac{k_{w}}{m}}+e_{a_{z}},
$$

where $\mathrm{p}$ is the differential operator. The noise term $e_{a_{z}}$ might be colored and correlated to the inputs $u_{i n 1}$ and $u_{i n 2}$ due to the closed-loop control. The model is discretized using the bilinear transformation $\mathrm{p}=\frac{2(\mathrm{q}-1)}{T_{s}(\mathbf{q}+1)}$ which gives

$$
\begin{aligned}
a_{z}= & \frac{2(\mathrm{q}-1)}{\left(2+\frac{k_{w}}{m} T_{S}\right) \mathrm{q}-\left(2-\frac{k_{w}}{m} T_{S}\right)}\left(\frac{k_{1}}{m} u_{i n 1}+\frac{k_{2}}{m} u_{i n 2}\right)+ \\
& \frac{\frac{k_{w}}{m} T_{S}(\mathrm{q}+1)}{\left(2+\frac{k_{w}}{m} T_{S}\right) \mathrm{q}-\left(2-\frac{k_{w}}{m} T_{S}\right)} g+v_{t},
\end{aligned}
$$

where $v_{t}$ is noise, $\mathrm{q}$ is the forward shift operator and $T_{s}$ is the sampling time. The discrete time model can now be rewritten in a regression form as

$$
a_{z}(t)=\varphi_{t}^{T} \vartheta+v_{t}
$$

where $\varphi_{t}=\left[-a_{z}(t-1), u_{i n 1}(t)-u_{i n 1}(t-1), u_{i n 2}(t)-u_{\text {in } 2}(t-\right.$ $1), g]$. The parameter vector $\vartheta=\left[\alpha, \beta_{1}, \beta_{2}, \beta_{3}\right]^{T}$ is given by

$\alpha=\frac{2-\frac{k_{w}}{m} T_{s}}{2+\frac{k_{w}}{m} T_{s}}, \beta_{1}=\frac{2 \frac{k_{1}}{m}}{2+\frac{k_{w}}{m} T_{s}}, \beta_{2}=\frac{2 \frac{k_{2}}{m}}{2+\frac{k_{w}}{m} T_{s}}, \beta_{3}=\frac{2 \frac{k_{w}}{m} T_{s}}{2+\frac{k_{w}}{m} T_{s}}$.

During the mission of the quadcopter, any changes of the battery's state of charge and the vertical aerodynamic drag are reflected via the parameters $\vartheta_{c}=\left[k_{1}, k_{2}, k_{w}\right]^{T}$. An estimate of $\vartheta_{c}$ can be obtained from the estimate of the discrete-time parameters $\vartheta_{d}=\left[\alpha, \beta_{1}, \beta_{2}\right]^{T}$ by solving

$$
\hat{\vartheta}_{c}=\text { solve }\left\{\hat{\vartheta}_{d}-\vartheta_{d}\left(\vartheta_{c}\right)=0\right\} \text {. }
$$

The estimated covariance of $\hat{\vartheta}_{c}$ can be derived by using Gauss approximation formula [13] and is given by

$$
P_{\hat{\vartheta}_{c}}=\left.\left[\frac{\partial \vartheta_{d}^{T}}{\partial \vartheta_{c}} P_{\hat{\vartheta}_{d}}^{-1} \frac{\partial \vartheta_{d}}{\partial \vartheta_{c}}\right]^{-1}\right|_{\vartheta_{c}=\hat{\vartheta}_{c}}
$$

\section{ESTIMATION OF THE VERTICAL MODEL}

\section{A. Least-squares estimation}

The parameters of the discrete-time model (12) can be estimated using the least squares (LS) method as given by

$$
\hat{\vartheta}_{L S}=\underset{\vartheta}{\arg \min } \frac{1}{N} \sum_{t=1}^{N}\left\|a_{z}(t)-\varphi_{t}^{T} \vartheta\right\|_{2}^{2},
$$

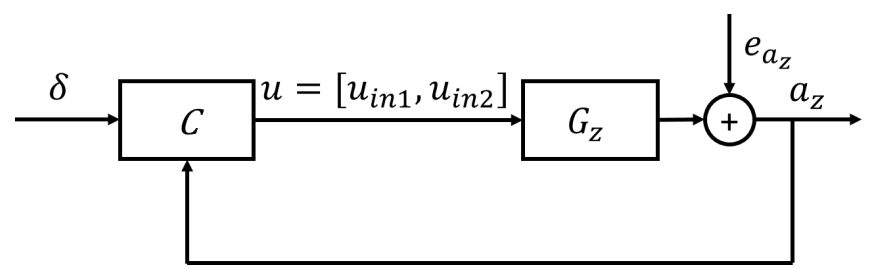

Fig. 2. The closed-loop block diagram of the system (10). The input $u=$ [ $\left.u_{i n 1}, u_{i n 2}\right]$ is computed using the $u_{\mathrm{ci}}$ signals and the output $a_{z}$ is measured with noise using IMU sensor. The noise $e_{a_{z}}$ is correlated with $u_{\mathrm{ci}}$ due to the effect of the feedback but is uncorrelated with the reference signal $\delta$.

which has the analytical solution

$$
\begin{aligned}
\hat{\vartheta}_{L S} & =\left[\frac{1}{N} \sum_{t=1}^{N} \varphi_{t} \varphi_{t}^{T}\right]^{-1} \frac{1}{N} \sum_{t=1}^{N} \varphi_{t} a_{z}(t) \\
& =R_{\varphi \varphi}^{-1} f_{\varphi a_{z} .}
\end{aligned}
$$

The estimated covariance matrix of the estimated parameters is given by

$$
P_{L S}=\hat{\sigma}^{2} R_{\varphi \varphi}^{-1}
$$

where $\hat{\sigma}$ is the estimated standard deviation of the residual.

\section{B. Instrumental variable method}

As mentioned in the Sec. II, the noise in the vertical model (12) might be colored and correlated with the inputs due to the closed-loop control. To overcome this difficulty, the instrumental variable (IV) method is used to estimate the parameters of (10). It is based on the use of an instrument vector $\xi(t)$ to extract the interesting information from $u_{\text {in } 1}, u_{\text {in } 2}$, and $a_{z}$.

In this paper, an extended IV method is used, where an estimate of the parameter vector $\vartheta$ is obtained by solving

$$
\hat{\vartheta}_{I V}=\underset{\vartheta}{\arg \min } \frac{1}{N} \sum_{t=1}^{N}\left\|\xi(t) L(\mathrm{q}) a_{z}(t)-\xi(t) L(\mathrm{q}) \varphi_{t}^{T} \vartheta\right\|_{Q}^{2},
$$

where $\|x\|_{Q}^{2}=x^{T} Q x, Q \geq 0$ is a weighting matrix and $L(\mathrm{q})$ is a stable prefilter.

In principle, the instruments $\xi(t)$ are created as

$$
\begin{aligned}
\xi(t)= & L(\mathrm{q})\left[\hat{a}_{z}^{0}(t), \cdots \hat{a}_{z}^{0}\left(t-n_{a z}\right), \hat{u}_{i n 1}^{0}(t), \cdots \hat{u}_{i n 1}^{0}\left(t-n_{u 1}\right), \cdots\right. \\
& \left.\hat{u}_{i n 2}^{0}(t), \cdots \hat{u}_{i n 2}^{0}\left(t-n_{u 2}\right), g\right]^{T},
\end{aligned}
$$

in which $\hat{u}_{i n 1}^{0}, \hat{u}_{i n 2}^{0}(t)$ and $\hat{a}_{z}^{0}(t)$ are the simulated inputs and output, respectively. These simulated signals are created using the reference signal $\delta(t)$ as

$$
\begin{aligned}
\hat{u}_{i n 1}^{0}(t) & =\hat{G}_{\delta u 1}(\mathrm{q}, \hat{\vartheta}) \delta(t) \\
\hat{u}_{i n 2}^{0}(t) & =\hat{G}_{\delta u 2}(\mathrm{q}, \hat{\vartheta}) \delta(t) \\
\hat{a}_{z}^{0}(t) & =\hat{G}_{\delta a z}(\mathrm{q}, \hat{\vartheta}) \delta(t),
\end{aligned}
$$

where $\hat{G}_{\delta u i}(\mathrm{q}, \hat{\vartheta})$ and $\hat{G}_{\delta a z}(\mathrm{q}, \hat{\vartheta})$ are estimated transfer functions from $\delta(t)$ to $u_{i n 1}(t), u_{i n 2}(t)$ and $a_{z}(t)$, respectively. This is illustrated in Fig. 2. However, since the true controller and the system are unknown, high-order black-box models are used to estimate these transfer functions to make sure that these models can capture the essential parts of the system dynamics. 


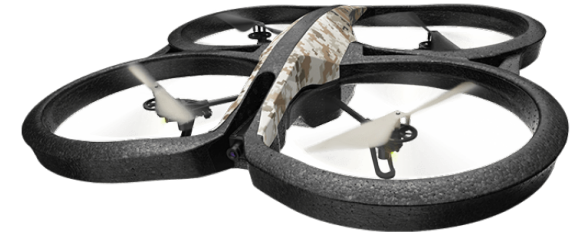

Fig. 3. AR Drone.

Another important issue in the IV method is to choose the prefilter $L(\mathrm{q})$ which has a considerable effect on the covariance of the estimated parameters [13]. If the true noise model structure is known, the covariance of the estimate can be minimized. More precisely, several choices of the prefilter are given in [17], which gives the optimal instruments and approximate optimal instruments for a closed-loop system. In this work, an ARMA noise model is estimated using the residual $v_{t}$ as the output and the inverse of the estimated noise model is used as the prefilter $L(\mathrm{q})$. Furthermore, the estimated covariance matrix $P_{\mathrm{iv}}$ is given by

$$
P_{\mathrm{iv}}=\hat{\sigma}^{2}\left(R_{\xi \bar{\varphi}}^{T} Q R_{\xi \bar{\varphi}}\right)^{-1} R_{\xi \bar{\varphi}}^{T} Q R_{\xi \xi} Q R_{\xi \bar{\varphi}}\left(R_{\xi \bar{\varphi}}^{T} Q R_{\xi \bar{\varphi}}\right)^{-1},
$$

where $R_{\xi \bar{\varphi}}=\mathbb{E}\left[\xi(t) \bar{\varphi}_{t}^{T}\right]=\mathbb{E}\left[\xi(t) L(\mathrm{q}) \varphi_{t}^{T}\right], \quad R_{\xi \xi}=$ $\mathbb{E}\left[\xi(t) \xi(t)^{T}\right]$ and $\hat{\sigma}$ is the estimated standard deviation of the residual. The IV algorithm implemented in this paper is similar to the one in [18].

\section{Experimental result}

Several experiments with vertical flight maneuvers have been carried out with an AR Drone quadcopter shown in Fig. 3. The IMU measurements and calculated rotor control signals are streamed wirelessly from AR Drone to a ground computer via Wi-Fi connection. This ground computer sent the pilot command to control the quadcopter to perform a vertical flight with increasing/decreasing altitude, zero roll/pitch and constant yaw angles. All maneuvers are carried out indoors in order to reduce the effect of the turbulence to the quadcopter's states. In detail, the first seconds of vertical acceleration measurements are used to estimate the bias of the IMU when the AR Drone is stationary. In total nine datasets have been collected, three datasets each for the nominal mass $455 \mathrm{~g}$, the mass $530 \mathrm{~g}$, and $586 \mathrm{~g}$. Fig. 4 shows a typical dataset collected in a particular experiment. In the figure, the bias estimates are already subtracted from the measurements.

For each mass case, three datasets have been used simultaneously to estimate the parameters of the standard or refined models. Tables II and III show the results using the LS and IV methods for two models, respectively. In detail, for the standard model in Table II, both methods give highly uncertain parameter estimates.

The estimated parameters with standard deviations of the refined model are shown in Table III. The LS method still gives very large estimated standard deviations that indicates
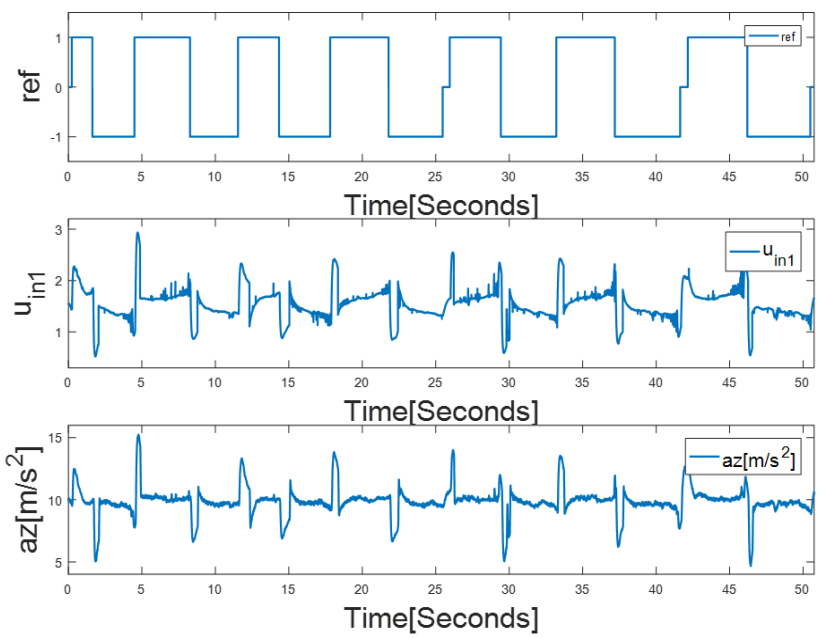

Fig. 4. An example of an experimental dataset. The first subplot shows the pilot command while $u_{i n 1}$ and $a_{z}$ are shown in the second and third subplots, respectively. The $u_{i n 1}$ is already normalized with factor $1 / 255^{2}$ since the maximum value of $u_{\mathrm{ci}}$ is 255 ( 8 bits memory [0-255]).

TABLE II

ESTIMATED CONTINUOUS-TIME PARAMETERS $k_{w}$ AND $k_{1}$ AND THEIR ESTIMATED STANDARD DEVIATIONS IN THE STANDARD MODEL USING LS AND IV METHODS FOR THE THREE SETS OF DATA WITH DIFFERENT MASSES.

\begin{tabular}{|c|c|r|r|r|}
\hline \multicolumn{2}{|c|}{ Param } & Mass 455 $g$ & Mass 530 $g$ & Mass 586 $g$ \\
\hline \multirow{2}{*}{$k_{w}$} & LS & $0.2588 \pm 0.0870$ & $0.3088 \pm 0.1784$ & $0.1812 \pm 0.1477$ \\
\cline { 2 - 5 } & IV & $0.3040 \pm 0.1340$ & $0.4051 \pm 0.0573$ & $0.3121 \pm 0.1047$ \\
\hline \multirow{2}{*}{$k_{1}$} & LS & $0.0437 \pm 0.0380$ & $0.0295 \pm 0.0651$ & $0.0612 \pm 0.0525$ \\
\cline { 2 - 5 } & IV & $2.8344 \pm 0.0148$ & $2.6975 \pm 0.0015$ & $2.5099 \pm 0.0039$ \\
\hline
\end{tabular}

that the parameter values are unreliable. On the other hand, the IV estimates vary less between the datasets and the variations seem to match the estimated standard deviations. Hence, the IV method seems like a promising approach to estimate the parameters of the refined model in this closedloop setup.

Fig. 5 shows the simulated vertical acceleration using the estimates of the standard and refined models obtained from the IV method for one typical validation dataset. The estimated parameters $\vartheta=\left[\alpha, \beta_{1}, \beta_{2}, \beta_{3}\right]^{T}$ obtained from the third set of data $(586 \mathrm{~g})$ are used to computed the coefficients of the discrete-time model associated to the first set of data $(455 \mathrm{~g})$, taking into account the mass difference. According to Fig. 5, the refined model $(57.10 \%$ model fit) gives a more accurate estimate of the vertical dynamics of the quadcopter than the standard model $(33.30 \%$ model fit). Note that the result is selected among the best for both models using only one dataset. More detailed results can be seen in Table IV where the value in each row in the third or fourth columns shows the average of the fitting percentages for three validation datasets $m_{v a l}$. It is clear that the refined model can capture the dynamics of the quadcopter better in all cases compared to the standard model. 
TABLE III

ESTIMATED CONTINUOUS-TIME PARAMETERS $k_{w}, k_{1}$ AND $k_{2}$ AND THEIR ESTIMATED STANDARD DEVIATIONS IN THE REFINED MODEL USING LS AND IV METHODS FOR THE THREE SETS OF DATA WITH DIFFERENT MASSES.

\begin{tabular}{|c|r|r|r|r|}
\hline \multicolumn{2}{|c|}{ Param } & Mass 455 $g$ & Mass 530 $g$ & Mass 586g \\
\hline \multirow{2}{*}{$k_{w}$} & LS & $0.2590 \pm 0.0848$ & $0.3068 \pm 0.1475$ & $0.1713 \pm 0.1473$ \\
\cline { 2 - 5 } & IV & $0.3040 \pm 0.0063$ & $0.2904 \pm 0.0083$ & $0.3052 \pm 0.0022$ \\
\hline \multirow{2}{*}{$k_{1}$} & LS & $0.1217 \pm 0.1298$ & $-0.1067 \pm 0.3205$ & $0.4957 \pm 0.2078$ \\
\cline { 2 - 5 } & IV & $0.5198 \pm 0.0482$ & $0.5165 \pm 0.0833$ & $0.4921 \pm 0.0217$ \\
\hline \multirow{2}{*}{$k_{2}$} & LS & $-0.0988 \pm 0.1248$ & $0.1870 \pm 0.2326$ & $-0.6443 \pm 0.1893$ \\
\cline { 2 - 5 } & IV & $1.5115 \pm 0.0305$ & $1.5574 \pm 0.0565$ & $1.5247 \pm 0.0171$ \\
\hline
\end{tabular}

TABLE IV

MODEL FIT VALUES FOR VALIDATION DATA. THE ESTIMATED PARAMETERS OBTAINED FROM THE IV METHOD USING $m_{\text {ref }}$ ARE PROJECTED TO THOSE USING $m_{v a l}$ FOR BOTH MODELS AND THREE SETS OF DATA $m_{v a l}$ ARE USED FOR VALIDATION. THE VALUES ON EACH ROW IN THE THIRD AND FOURTH COLUMNS SHOW THE AVERAGE FITTING PERCENTAGES FOR THREE SETS OF DATA $m_{v a l}$ OF THE STANDARD AND REFINED MODELS, RESPECTIVELY.

\begin{tabular}{|c|c|r|r|}
\hline$m_{\text {ref }}$ & $m_{\text {val }}$ & Standard model & Refined model \\
\hline \multirow{2}{*}{$455 g$} & $530 g$ & $8.83 \%$ & $56.62 \%$ \\
\cline { 2 - 4 } & $586 g$ & $-5.69 \%$ & $55.90 \%$ \\
\hline \multirow{2}{*}{$530 g$} & $455 g$ & $22.54 \%$ & $56.02 \%$ \\
\cline { 2 - 4 } & $586 g$ & $5.71 \%$ & $55.80 \%$ \\
\hline \multirow{2}{*}{$586 g$} & $455 g$ & $31.71 \%$ & $56.95 \%$ \\
\cline { 2 - 4 } & $530 g$ & $26.31 \%$ & $56.89 \%$ \\
\hline
\end{tabular}

\section{MAss estimation}

\section{A. Using the vertical dynamic model}

If a model has been estimated from a reference dataset where the mass is known, the system identification approach from the previous section can be used to monitor changes in the mass. Table $\mathrm{V}$ shows the estimates of the masses using both the standard and refined models. In fact, six mass estimation setups are obtained using three datasets corresponding to three different masses of the quadcopter. For each combination, a mass estimate $m_{c}$ could be obtained

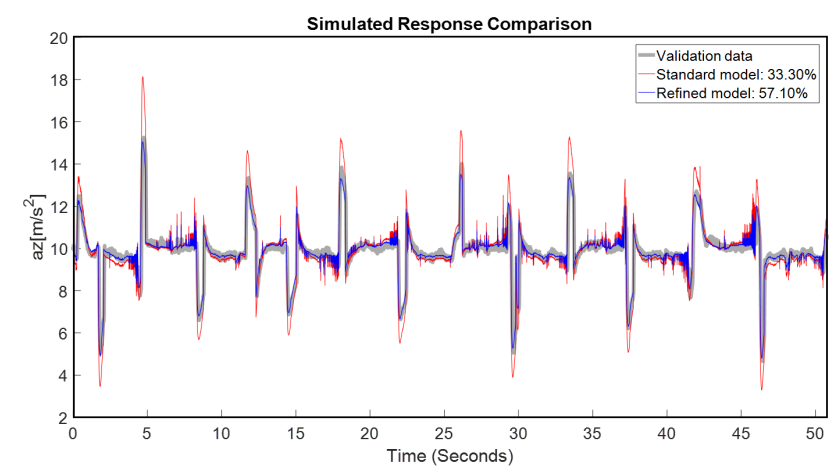

Fig. 5. Measured (grey) and simulated outputs from the standard model (solid red) and refined model (solid blue).
TABLE V

THE AVERAGE ESTIMATES OF THE MASSES USING THE RATIOS $\frac{k_{w}}{m}, \frac{k_{1}}{m}$ AND $\frac{k_{2}}{m}$. THE MASS ESTIMATE $\hat{m}_{c}$ IS THE AVERAGE OF THREE ESTIMATES OBTAINED FROM THREE RATIOS FOR THE REFINED MODEL AND TWO RATIOS FOR STANDARD MODEL.

\begin{tabular}{|c|r|r|r|}
\hline$m_{r e f}$ & $m_{c}$ & $\hat{m}_{c}$ (Standard) & $\hat{m}_{c}$ (Refined) \\
\hline \multirow{2}{*}{$455 g$} & $530 g$ & $596.1 g$ & $526.3 g$ \\
\cline { 2 - 4 } & $586 g$ & $560.3 g$ & $578.1 g$ \\
\hline \multirow{2}{*}{$530 g$} & $455 g$ & $419.1 g$ & $458.6 g$ \\
\cline { 2 - 4 } & $586 g$ & $508.9 g$ & $582.6 g$ \\
\hline \multirow{2}{*}{$586 g$} & $455 g$ & $478.5 g$ & $461.6 g$ \\
\cline { 2 - 4 } & $530 g$ & $618.2 g$ & $534.1 g$ \\
\hline
\end{tabular}

TABLE VI

THE RELATIONS OF THE SYSTEM CHANGES AND THE RATIOS $\frac{k_{w}}{m}, \frac{k_{1}}{m}$ AND $\frac{k_{2}}{m}$. THE TWO RATIOS $\frac{k_{1}}{m}$ AND $\frac{k_{2}}{m}$ VARY SIMILARLY WITH RESPECT TO DIFFERENT FAULT/CHANGE SCENARIOS.

\begin{tabular}{|r|r|r|r|}
\hline Ratio & Battery efficiency $\uparrow$ & Drag parameter $\uparrow$ & Mass variation $\uparrow$ \\
\hline$\frac{k_{w}}{m}$ & - & $\uparrow$ & $\downarrow$ \\
\hline$\frac{k_{1}}{m}$ & $\downarrow$ & - & $\downarrow$ \\
\hline$\frac{k_{2}}{m}$ & $\downarrow$ & - & $\downarrow$ \\
\hline
\end{tabular}

using estimates of two ratios of $\beta_{1, \text { ref }}=\frac{k_{1, r e f}}{m_{\text {ref }}}$ and $\beta_{1, c}=\frac{k_{1, c}}{m_{c}}$. With a known $m_{r e f}$, the mass $\hat{m}_{c}$ is estimated as $\hat{m}_{c}=\frac{\hat{k}_{1, r e f}}{\hat{\beta}_{1, c}}$ where $\hat{k}_{1, \text { ref }}$ and $\hat{\beta}_{1, c}$ are the estimated values of $k_{1, \text { ref }}$ and $\beta_{1, c}=\frac{k_{1, c}}{m_{c}}$, respectively. Performing similar calculations for the ratios $\alpha$ and $\beta_{2}$ gives three mass estimates. These mass estimates will be averaged to achieve a combined mass estimate. As Table $\mathrm{V}$ shows, the refined model provides more accurate mass estimates than the standard model in all combinations.

\section{B. Comparison with the horizontal dynamic model}

An alternative approach to estimation of the mass change of a quadcopter is to use a lateral dynamic model. During a flight mission, there could be intervals where the quadcopter's movements are either dominantly horizontal or vertical. Hence, it could be interesting to be able to estimate the mass from both horizontal and vertical excitations. In [12], an estimator has been designed to estimate the change of the mass of the quadcopter from horizontal movements. It is shown that the mass estimate error is about $8 \mathrm{~g}$ for all different experimental mass setups. Moreover, it can be seen from Table $\mathrm{V}$ that the use of the estimator based on the refined vertical model provides an estimate with similar accuracy. Hence, these two estimators could be used in combination to estimate changes in the mass of the quadcopter during a large part of the flight maneuver.

\section{DIAGNOSIS AND DISCUSSION}

Any fault representing malfunctioning of a component of the quadcopter, for example as a result a locked actuator, affects the performance of the overall system. The fault has to be estimated using fault detection and isolation (FDI) 
and then fault tolerant control (FTC) can be used to retain the quadcopter's maneuverability. In particular, with respect to the refined model, there are three different fault/change scenarios that could be investigated as shown in Table VI and as described as follows:

- The first fault/change scenario may be the decrease of the battery voltage. In detail, the thrust coefficients $k_{1}$ and $k_{2}$ depend on the battery characteristics. The longer the flight time is, the more the battery state of charge decreases, which will result in a decrease of the ratios $\frac{k_{1}}{m}$ and $\frac{k_{2}}{m}$. On the other hand, the drag ratio $\frac{k_{w}}{m}$ will typically not be affected. An estimator could then be designed to monitor the state of the battery to alert the quadcopter to land automatically before the battery is completely drained.

- If the quadcopter carries objects with significant size between different waypoints, the dynamic model of the quadcopter might be changed. One possibility is that the two ratios $\frac{k_{1}}{m}$ and $\frac{k_{2}}{m}$ remain constant while the ratio $\frac{k_{w}}{m}$ varies. This should also be possible to detect and the controller can then use the knowledge of the parameter change to adapt the overall system's behavior.

- It can be seen that all three ratios $\frac{k_{1}}{m}, \frac{k_{2}}{m}$ and $\frac{k_{w}}{m}$ vary similarly when the mass of the quadcopter is changed. Hence, mass changes during and between missions should be possible to detect.

It is also possible to consider combinations of the scenarios mentioned above. In this case, it might be necessary to combine several approaches, e.g., the vertical modeling approach presented here and the horizontal modeling approach from [12].

\section{CONCLUSIONS}

In this work, we have dealt with the aerodynamic modeling problem in terms of the vertical dynamics of a quadcopter. It has been shown that a model based on a refined thrust equation can capture the system dynamics better than a standard model and that the model parameters can be estimated accurately from experimental closed-loop data using an IV approach. Furthermore, we have discussed how this system identification approach can be used to monitor changes in the mass of the quadcopter and the possibilities to detect also other faults or changes in the system.

Future work includes further development of the diagnosis functionality outlined here, the combination of several modeling approaches and the extension of the current framework to fixed-wing UAVs.

\section{ACKNOWLEDGMENT}

This project has received funding from the European Union's Horizon 2020 research and innovation programme under the Marie Sklodowska-Curie grant agreement No. 642153 .

\section{REFERENCES}

[1] G. Cai, B. M. Chen, and T. H. Lee, "An overview on development of miniature unmanned rotorcraft systems," Frontiers of Electrical and Electronic Engineering in China, vol. 5, no. 1, pp. 1-14, 2010.
[2] F. Fraundorfer, L. Heng, D. Honegger, G. H. Lee, L. Meier, P. Tanskanen, and M. Pollefeys, "Vision-based autonomous mapping and exploration using a quadrotor MAV," in 2012 IEEE/RSJ International Conference on Intelligent Robots and Systems, Vilamoura, Algarve, Portugal, Oct 2012, pp. 4557-4564.

[3] C. Bills, J. Chen, and A. Saxena, "Autonomous MAV flight in indoor environments using single image perspective cues," in 2011 IEEE International Conference on Robotics and Automation, Shanghai, China, May 2011, pp. 5776-5783.

[4] M. D. Hua, T. Hamel, P. Morin, and C. Samson, "Introduction to feedback control of underactuated VTOL vehicles: A review of basic control design ideas and principles," IEEE Control Systems, vol. 33, no. 1, pp. 61-75, Feb 2013.

[5] R. Mahony, V. Kumar, and P. Corke, "Multirotor aerial vehicles: Modeling, estimation, and control of quadrotor," IEEE Robotics Automation Magazine, vol. 19, no. 3, pp. 20-32, Sept 2012.

[6] S. Lupashin, A. Schollig, M. Sherback, and R. D'Andrea, "A simple learning strategy for high-speed quadrocopter multi-flips," in 2010 IEEE International Conference on Robotics and Automation, May 2010, pp. 1642-1648.

[7] H. T. Dinh, M. H. Cruz Torres, and T. Holvoet, "Dancing UAVs: Using linear programming to model movement behavior with safety requirements," in 2017 International Conference on Unmanned Aircraft Systems, Miami, Florida, USA, June 2017.

[8] P. Martin and E. Salaun, "The true role of accelerometer feedback in quadrotor control," in Proceedings for the 2010 IEEE International Conference on Robotics and Automation, Anchorage, USA, 2010, pp. 1623-1629.

[9] P.-J. Bristeau, P. Martin, E. Salaun, and N. Petit, "The role of propeller aerodynamics in the model of a quadrotor uav," in In proceedings for the European Control Conference 2009, vol. 1-6, Budapest, Czech, 2009, pp. 683-688.

[10] F. Gustafsson, Adaptive filtering and change detection. Wiley Online Library, 2000.

[11] D. Rupp, G. Ducard, E. Shafai, and H. P. Geering, "Extended multiple model adaptive estimation for the detection of sensor and actuator faults," in Proceedings of the 44th IEEE Conference on Decision and Control, Dec 2005, pp. 3079-3084.

[12] D. Ho, J. Linder, G. Hendeby, and M. Enqvist, "Mass estimation of a quadcopter using IMU data," in 2017 International Conference on Unmanned Aircraft Systems, Miami, Florida, USA, June 13-16 2017.

[13] L. Ljung, System identification - Theory for the User, 2nd ed. Prentice-Hall, 1999.

[14] M. B. Tischler and R. K. Remple, Aircraft and rotorcraft system identification. AIAA education series, 2006.

[15] J. Svacha, K. Mohta, and V. Kumar, "Improving quadrotor trajectory tracking by compensating for aerodynamic effects," in 2017 International Conference on Unmanned Aircraft Systems, Miami, Florida, USA, June 2017.

[16] G. M. Hoffmann, H. Huang, S. L. Wasl, and E. C. J. Tomlin, "Quadrotor helicopter flight dynamics and control: Theory and experiment," in In Proc. of the AIAA Guidance, Navigation, and Control Conference, 2007.

[17] M. Gilson, H. Garnier, P. Y. Young, and P. M. J. Van den Hof, "Optimal instrumental variable method for closed-loop identification," IET Control Theory \& Applications, vol. 5, no. 10, pp. 1147-1154, 2011.

[18] J. Linder, M. Enqvist, and F. Gustafsson, “A Closed-loop Instrumental Variable Approach to Mass and Center of Mass Estimation Using IMU Data," in 53rd IEEE Conference on Decision and Control, Los Angeles, California, USA, December 2014. 